\title{
Chronology of Levinas's Talmudic Lectures at the Colloque des intellectuels juifs de langue française
}

4 MAY 1957 First Colloque des intellectuels juifs de langue française. No lecture by Levinas.

22 SEPTEM BER 1959 Second Colloque des intellectuels juifs de langue française, "Entre deux mondes: Biographie spirituelle de Franz Rosenzweig," in E. Amado Lévy-Valensi and J. Halpérin, La conscience juive: Données et débats, PUF, 1963.

25 SEPTEM BER 1960 Third Colloque des intellectuels juifs de langue française, "Temps messianiques et temps historiques dans le chapitre XI du 'Traité Sanhédrin'” (Sanhedrin 99a), in La conscience juive: Données et débats, PUF, 1963.

8 OСтОв R 1961 Fourth Colloque des intellectuels juifs de langue française, "Le messianisme d'après un texte talmudique" (Sanhedrin 98b-99a), in E. Amado Lévy-Valensi and J. Halpérin, La conscience juive face à l'Histoire: Le pardon, PUF, I965.

13-14 ОСтОВЕR 1963 Fifth Colloque des intellectuels juifs de langue française, "Envers autrui" (Yoma 87a), in E. Amado Lévy-Valensi and J. Halpérin, La conscience juive face à l'Histoire: Le pardon, PUF, 1965.

11-12 ОСтовеR 1964 Sixth Colloque des intellectuels juifs de langue 
française, "La tentation de la tentation" (Shabbat 88a-b), in E. Amado Lévy-Valensi and J. Halpérin, Tentations et actions de la conscience juive, PUF, I97I.

24-25 Остов в 1965 Seventh Colloque des intellectuels juifs de langue française, "Terre promise et terre premise" (Sota 34b-35a), in E. Amado Lévy-Valensi and J. Halpérin, Israël dans la conscience juive: Données et débats, PUF, I97I.

9-10 Остов в 1966 Eighth Colloque des intellectuels juifs de langue française, "Vieux comme le monde?" (Sanhedrin 36b-37a), in E. Amado Lévy-Valensi and J. Halpérin, Tentations et actions de la conscience juive, PUF, I97I.

JANUARY 1968 Ninth Colloque des intellectuels juifs de langue française, "Israel" Levinas is absent.

16, 17, 18, 19 MARCH 1969 Tenth Colloque des intellectuels juifs de langue française, "Judaïsme et revolution" (Baba Metsia 83a-b), in J. Halpérin and G. Lévitte, Jeunesse et revolution. La Conscience juive: Données et débats, PUF, 1972.

25, 26 остовеR 1970 Eleventh Colloque des intellectuels juifs de langue française, "Jeunesse d'Israël” (Nazir 66a-b), in J. Halpérin and G. Lévitte, Jeunesse et révolution dans la conscience juive: Données et débats, PUF, 1972.

31 OCtOBER, 1 NOVEMBER 1971 Twelfth Colloque des intellectuels juifs de langue française, "Désacralisation et désensorcellement" (Sanhedrin 67a-b), in J. Halpérin, Lautre dans la conscience juive (suivi de Le sacré et le couple), PUF, I973.

15-16 ОСтовеR 1972 Thirteenth Colloque des intellectuels juifs de langue française, "Et Dieu créa la femme" (Berakhot 6ra), in J. Halpérin, L'autre dans la conscience juive (suivi de Le sacré et le couple), PUF, I973.

NOVEM BER 1973 Fourteenth Colloque des intellectuels juifs de langue française, "Sabbath." Emmanuel Levinas is absent.

10, 11 NOVEM BER 1974 Fifteenth Colloque des intellectuels juifs de langue 
française, "La solitude de Kippour" (Makoth 23a-b), in J. Halpérin and G. Lévitte, Solitude d'Israël: Données et débats, PUF, 1975.

9 NOVEMBER 1975 Sixteenth Colloque des intellectuels juifs de langue française, "Les dommages causés par le feu" (Baba Kima 6oa-b), in J. Halpérin and G. Lévitte, La conscience juive face à la guerre, PUF, 1976.

27, 28, 29 NOVEMBER 1976 Seventeenth Colloque des intellectuels juifs de langue française, "Modèles de la permanence" (Menahot 99b-Iooa), in J. Halpérin and G. Lévitte, Le modèle de l'Occident, PUF, 1977.

Остов 1977 Eighteenth Colloque des intellectuels juifs de langue française, "The Muslim Community." Levinas is absent.

25, 26, 27 NOVEM BER 1978 Nineteenth Colloque des intellectuels juifs de langue française, "Les villes-refuges" (Makot IOa), in J. Halpérin and G. Levitte, Jérusalem, l'Unique et l’Universelle, PUF, I979.

24, 25, 26 NOVEM BER 1979 Twentieth Colloque des intellectuels juifs de langue française, "Qui joue le dernier ?" (Yoma Ioa), in J. Halpérin and G. Levitte, Politique et religion: Données et débats, Gallimard Idées, 198I.

1980 Twenty-First Colloque des intellectuels juifs de langue française, "Le Pacte" (Sota 37a-b), in L’au delá du verset, Les Éditions de Minuit, 1982.

28, 29, 30 NOVEMBER 1981 Twenty-Second Colloque des intellectuels juifs de langue française, "Pour une place dans la Bible" (Megillah 7a), in J. Halpérin and G. Levitte, La Bible au présent, Gallimard Idées, 1982.

24, 25 APRIL 1983 Twenty-Third Colloque des intellectuels juifs de langue française, "The Translation of Scriptures" (Megillah 9a-b), in Israel le judaïsme et l'Europe, Gallimard Idées, 1984.

28, 29, 30 JANUARY 1984 Twenty-Fourth Colloque des intellectuels juifs de langue française, "Mépris de la Thora comme idolâtrie" (Sanhedrin 99a-b), in Idoles: Données et débats, Éditions Denoël, 1985.

DECEM BER 1984 Twenty-Fifth Colloque des intellectuels juifs de langue française, "Au-delà du souvenir" (Berakhot I2b-I3a), in J. Halpérin and 
G. Lévitte, Mémoires et histoire: Données et débats, Éditions Denoël, I986.

Twenty-Sixth Colloque des intellectuels juifs de langue française NO RECORD FOUND.

DECEM BER 1986 Twenty-Seventh Colloque des intellectuels juifs de langue française, "Les Nations et la présence d'Israël” (Pesachim Iı8b), in J. Halpérin and G. Lévitte, Les soixante-dix nations: Données et débats, Éditions Denoël, I987.

DECEM BER 1987 Twenty-Eighth Colloque des intellectuels juifs de langue française, "Socialité et argent," in J. Halpérin and G. Lévitte, L’Argent: Données et débats, Denoël, I989.

3, 4, 5 DECEM BER 1988 Twenty-Ninth Colloque des intellectuels juifs de langue française, “Au-dela de l'Etat dans l'Etat” (Tamid 3rb-32b), in J. Halpérin and G. Lévitte, La Question de l'état: Données et débats, Denoël, I989.

9, 10, 11 DECEMBER 1989 Thirtieth Colloque des intellectuels juifs de langue française, "Qui est soi-même?" (Chullin 88b-89a), in J. Halpérin and G. Lévitte, Le "quant-à-soi: Données et débats, Denoël, I99I. 
EMMANUEL LEVINAS'S TALMUDIC TURN 
This page intentionally left blank 